\title{
A collaborative approach to ROMP TEAP registrar training
}

\author{
Natalie Clements ${ }^{1} \cdot$ Ciara Mac Nally $^{2} \cdot$ Rebecca Moylan $^{3} \cdot$ David Waterhouse $^{4}$
}

Published online: 19 March 2021

(c) Australasian College of Physical Scientists and Engineers in Medicine 2021

\section{Introduction}

The Australasian College of Physical Scientists and Engineers in Medicine (ACPSEM) is responsible for administering the pathway to Certification for Radiation Oncology Medical Physics (ROMP) Registrars in Australia and New Zealand. ACPSEM have developed the Training Education and Assessment Program (TEAP) [1] which is undertaken by the ROMP Registrar at an ACPSEM accredited facility with several requirements the Registrar must complete to obtain Certification. Major requirements are successful completion of post-graduate study at an accredited institution, and a clinical Registrar placement held over several years in a clinical Department to complete a detailed list of competencies ranging from theoretical understanding to clinical project management across core and ancillary topics related to Medical Physics. With evaluation of the Registrar occurring progressively throughout the program via assessment of competence, written reports, written exam, and finally via practical and oral exam, TEAP aims to equip candidates with the broad range of theoretical, practical, and professional skills required to successfully attain Certification, but more importantly to operate safely and competently within the profession.

Based on the recommendations of the International Atomic Energy Agency for ROMP clinical training [2] the ACPSEM have developed a ROMP TEAP Clinical Training Guide (CTG) [3] which details the required Learning Outcomes (i.e. competencies) and associated Assessment

Natalie Clements

Natalie.Clements@genesiscare.com

1 GenesisCare, Frankston Private Hospital, Frankston, VIC, Australia

2 GenesisCare, Fiona Stanley Hospital, Murdoch, WA, Australia

3 GenesisCare, Lake Macquarie Private Hospital, Gateshead, NSW, Australia

4 GenesisCare, Wembley, WA, Australia
Criteria that are to be addressed by each Registrar throughout their program. The Learning Outcomes are separated into different levels of increasing complexity given the desired outcome required; Level 1, Level 2, Level 3. Level 1 generally covers understanding of fundamental physics concepts and the Registrar observing or doing various clinical tasks under close supervision, Level 2 progresses into the application of the learned physics concepts with minimal supervision, and Level 3 progresses further to an overarching understanding of systems or concepts with the Registrar taking on more of a management role in the task. It is the responsibility of the Registrar's employer to actively train the Registrar and to provide learning opportunities so they can meet all TEAP Assessment Criteria within their allocated timeframe. The content of the CTG is the same for all Registrars; therefore, between different institutions with different Registrars there is substantial duplication in training effort at the local level. At many institutions this training responsibility typically falls to a small group of individuals intimately aware of the CTG content and the ACPSEM's requirements of the Registrar. This small team includes but may not be limited to the Registrar's immediate Supervisor, Informal Trainers within the institution's Physics team, Clinical Preceptors (where applicable) or TEAP training coordinators. When the training commitment falls to a small group it can be challenging to provide regular and structured training opportunities, and to maintain these opportunities without cancellations when clinical demands increase. With ROMP TEAP now required to be completed within a 3 -year timeframe this adds substantial workload in terms of the provision of required learning opportunities and hence time commitment from qualified and experienced ROMPs, who often must balance the needs of a Registrar in training with the clinical needs of their Department or institution. The ad-hoc nature of unscheduled training can hinder progress and add to the pressure for completion as time progresses. The sentiments of Perkins in 2013 [4] regarding the demands of TEAP on departments and Registrars continue to be relevant today as the standards 
remain high, there is still a large number of competencies to address, and departments continue to learn to provide structured training programs around the challenges associated with high clinical workloads. Perkins [4] identifies the issues around timely completion of TEAP Registrars being attributable to these challenges as well as to a general lack of training support. To better support TEAP, departments therefore need to develop a well-structured program that frequently addresses TEAP requirements and is resilient to local staffing and clinical workload. A collective and collaborative approach to training multiple Registrars from multiple institutions can present many benefits including; more efficient use of educator's time, opportunities for Registrars to interact with ROMPs at sites other than their own, opportunities for ROMPs to focus their training effort in a specific area of expertise, reducing duplicated effort in training, easing the local training workload by sharing the responsibility with many qualified physicists, and providing frequent learning opportunities in a sustainable, consistent, and standardized manner.

At GenesisCare Australia we have designed and implemented a collaborative weekly TEAP scheme that leverages our expertise and experience, and our size, by involving all of our more than 60 qualified physicists within the national network to streamline learning opportunities for GenesisCare ROMP Registrars. This initiative aligns with the recommendations from the Royal Australian and New Zealand College of Radiologists of a networked approach to the training of TEAP Registrars in rural and remote settings [5]; although our program extends this approach to network all GenesisCare Registrars and Physicists at all metro and rural departments. Since May 2019 ROMP Registrars across GenesisCare Australia have received a range of weekly learning opportunities tailored to all levels of training from experts within their field. This implementation has addressed a number of aims including distributing the TEAP training responsibility to a wider group of qualified physicists to ease the local workload, training multiple Registrars simultaneously to reduce duplication of training, drawing topic material directly from the CTG, providing Registrars equal access to training regardless of their geographic location, and introducing Registrars to a wider network of other Registrars and qualified physicists from whom their training can benefit. Furthermore, the Registrar is made the active learner in the GenesisCare national program with the training style less rote-based and more interactive and collaborative, as supported by Wilfert in mentoring the successful TEAP Registrar [6]. Weekly sessions add an externally managed and facilitated TEAP activity to the Registrar's calendar which are in addition to their local site-based clinical training activities, and Registrars continue to report to their supervisor for feedback and assessment on the activities undertaken in the weekly sessions. Since the initial design and organizational stage, the GenesisCare program has undergone several internal audits and improvements to ensure Registrars gain maximum benefit from the training sessions.

We present an overview of the GenesisCare Australia program here to provide institutions currently training ROMP Registrars with a roadmap towards initiating a similar scheme at their own institution or in collaboration with multiple institutions - public and/or private. This overview details the design and development phase, the specifics of each session format, a discussion on the successes and lessons learned in the program, and considerations to be made when implementing a similar program.

\section{Designing the program}

The idea for a nationalized, collaborative approach to training ROMP TEAP Registrars was initiated in February 2019 by the WA Chief of Medical Physics, a Medical Physicist and TEAP Supervisor in WA, and a Medical Physicist and Clinical Preceptor in VIC. At the time of initiation GenesisCare Australia had approximately 55 qualified medical physicists employed across WA, SA, VIC, NSW, and QLD; the intent was that all physicists across the network would be involved to provide training to Registrars in topics aligned with their own expertise and interests. To provide a weekly session each physicist would need only facilitate a learning session approximately once in a calendar year, considerably reducing the local training workload, freeing time for competency assessments and practical learning supervision (for example). It was also anticipated that session cancellations would be infrequent as the ROMP physicist would have an increased sense of responsibility to deliver one learning session in a 12-month period with all GenesisCare Registrars in attendance. In the event of a cancellation there would still be a large pool of physicists to draw on to fill any gaps. The attraction for TEAP Registrars was a curated and regular set of training presentations and learning activities tailored to the CTG, each provided by a trainer with a particular interest in the subject at hand, and the opportunity to learn in collaboration with a study group of like-minded Registrars. Each manager was expected to maintain the Registrar's schedule free of clinical commitments at the nominated time each week to attend the online training session.

The design phase began in February 2019 and it was approximately 3 months before the program's first session in May 2019. The organizers aimed to generate a program that was driven by CTG Learning Outcomes that provided frequent opportunities for Registrars to address Assessment Criteria, and gave Registrars an educational forum in which to continually practice and refine verbalizing their medical physics knowledge - a key aspect of the ACPSEM's ROMP examination process. The organizing team solicited support 
from GenesisCare Australia's National Physics Director and all five state Chiefs of Medical Physics and met regularly to develop a weekly format and calendar of events. The weekly sessions were designed to have a variety of learning opportunities that addressed the aims of the program: fortnightly assignment-based tutorials, Registrar presentations, lectures by a qualified ROMP, and several event-specific sessions such as written exam preparation, and pre-conference poster/ oral presentation practice sessions. The weekly sessions were to be delivered over a videoconferencing tool that was available to all GenesisCare Australia employees. A blank calendar for the remainder of 2019 was generated in Excel that considered all state public holidays, major national/ international conferences, written exam dates, practical/ oral exam weekend sessions, and 4 weeks' break from midDecember to mid-January. Based on the calendar Thursdays were the optimal day to schedule a weekly session with the least disruptions due to public holidays. After consideration of the time differences between WA and eastern states, and states that observe daylight savings, a weekly start time of 1:30pm AEST was deemed optimal.

The fortnightly tutorials were divided amongst each state in which GenesisCare operates (WA, SA, VIC, NSW, QLD), and the weeks in the calendar were filled with open slots for fortnightly tutorials, Registrar presentations, and ROMP lectures. No weekly sessions were slated during major Australian conferences. The session immediately prior to TEAP written exam dates in March and September were allocated as an exam preparation Q\&A session for Registrars sitting the exams, and the session immediately following a written exam was set to enable Registrars who sat the exam to debrief and share the experience. The session prior to the ACPSEM's Engineering, Physicists and Scientists in Medicine (EPSM) conference in November was allocated as an open Registrar presentation session to practice posters or oral presentations.

The organizers developed separate written guidelines for Registrars and tutors. For tutors the guidelines explained the tutorial format, described how to develop a question list including examples, and the role and expectations of both the tutor and Registrar during the tutorial. The Registrar guidelines detailed the format of each session type and the role and expectation of the Registrar within each session. Other documentation developed included a list of competencies directly from CTG v3.6 (e.g. 3.5 In-vivo dosimetry, 4.2 MLC commissioning, etc.) that trainers could use as the basis of their topic selection and to track selections to minimise repeats, and an attendance spreadsheet. All documentation was kept on an online communal platform for all organizing team members to access and edit as needed. The organizers also created a database to store all material from the weekly sessions including lectures, tutorial question lists, expected answers, and answers submitted by Registrars.
These materials can be used with a new group of Registrars after 3-4 years has lapsed. This assists to avoid repetition for Registrars who may have already been exposed to the material, and to guide and assist trainers when developing their training materials.

The organizers then recruited individual ROMPs from across the network to fill the open sessions. For the tutorials and lectures that were to be hosted by qualified ROMPs, the Chief Medical Physicists were provided the calendar dates allocated to their state and requested to solicit nominations or appoint a physicist from within their team for each tutorial and lecture date by a specified deadline. The hosting physicists were requested to identify their tutorial topic ahead of time from the provided list of competencies, with the organizing team ensuring no duplication and that there was variety across CTG modules. For Registrar presentations the calendar dates were sent to all GenesisCare Registrars and each was requested to select one date to deliver a presentation on a first come first served basis. Topics were nominated closer to their presentation date. All tutorials were scheduled to run for $1.5 \mathrm{~h}$ and all lectures and Registrar presentations for $1 \mathrm{~h}$ including time for questions. Specific details on the format for tutorials, Registrar presentations, and ROMP lectures are provided here.

\section{Tutorials}

The basic premise of each tutorial was to have the Registrar be an active learner by giving them a set of questions to attempt and submit beforehand. The hosting qualified ROMP would then facilitate discussion as Registrars present and discuss their answers with the group. This teaching style is already being implemented by Universities in the context of graduate-level physics as outlined in Haworth et al. with a recent required shift to remote and digital learning as a result of COVID-19 [7]. This method of learning and teaching is aimed at better developing a Registrar's understanding of topic material with a focus on problem solving and independent thinking; skills required of medical physicists.

The hosting qualified physicist, or tutor, is responsible to develop a question list on their chosen topic, and the expected responses. Tutorial questions were selected to cover aspects of CTG Level 1, Level 2, and Level 3 for the related competency with all relevant CTG Learning Outcomes clearly identified for each question (e.g. 1.2.2.1, 3.3.3.1). Tutors are encouraged to use previous written exam questions for Level 2, and for Level 3 to develop clinical problem-solving style questions where the answer cannot be found in a textbook but requires deeper understanding of the broader impact of the issue at hand, and lateral thinking. The question list and expected responses are reviewed by the organizing team for clarity, spelling/ grammar, appropriateness, and length before the question 
list is distributed to the Registrars prior to the tutorial. Registrars are given 2 weeks to address the questions and must submit their answers to the tutor a few days prior to the tutorial for review. Registrars are requested to identify which of the listed relevant Learning Outcomes they have completed to guide the tutor in the ability of the Registrar. The tutor is not responsible for individually marking assignments but reviews the submitted responses for accuracy and common misconceptions or missing information. They then use that information to assist them to facilitate the Registrars to develop a comprehensive response to each question during the tutorial, and to provide necessary missing details that may be new learning for some of the participating Registrars.

During the tutorial the tutor facilitates discussion by selecting Registrars at random to discuss their submitted answers, providing feedback on the supplied answer, and elucidating a comprehensive answer. Tutors are requested to provide each Registrar who submitted answers an opportunity to present at least one of their responses. While it can be tempting to select the best response to each question, this can favour more senior or experienced Registrars and thus reduce opportunities for more junior or inexperienced Registrars to practice verbalizing medical physics concepts. By discussing a 'less complete' response, Registrars are encouraged to assess the information presented to them, identify missing information, and to share and justify their additions or corrections collaboratively.

After the tutorial the onus is on the Registrar to amend their responses based on the discussion during the tutorial, and have their local Supervisor review their answer for use as TEAP evidence. The expected answers developed by the tutor are sent to all Supervisors to guide them in their assessment of their Registrar's responses, and Registrars are welcome to contact the tutor for any further clarification.

At the outset, tutorials in 2019 were divided amongst the five states in which GenesisCare operates based on the number of Registrars in each state. Upon reflection this resulted in an unequal distribution of tutorials per state; furthermore, NSW had no Registrars in 2019 but still wished to participate in the scheme. Given the network of physicists is ultimately responsible for delivering tutorials regardless of the number of Registrars in their state or centre, in 2020 the tutorials were allocated based on the number of qualified physicists within each state. This resulted in a more equitable distribution of tutorials for each state to facilitate within the 12-month timeframe: QLD:6, VIC:6, NSW:6, WA:4, SA:4. and also equally distributed access to continued professional development (CPD) activities that ROMPs within each state could use to assist them to maintain their own ACPSEM Registration.

\section{Registrar presentations}

Registrar presentations occur approximately once per month, with Registrars selecting their rostered date in the calendar year on a first come first served basis. With approximately 10 Registrars at any one time enrolled in the TEAP program at GenesisCare each Registrar presents once in a calendar year. Dates towards the end of the year are reserved for Registrars that may join TEAP after the national calendar is finalized.

These presentations are a requirement and give Registrars an opportunity to practice their presentation skills, build confidence in explaining complex medical physics concepts in front of their qualified peers, and receive valuable feedback. Registrars are encouraged to present on items that may assist them to achieve completion of a Learning Outcome, on clinical tasks they are involved in or leading, research project work, or their best works or progressive reports. Registrars are requested to discuss their presentation topic with their Supervisor prior to their allocated time, and to be prepared to present on their nominated date. An invitation for the videoconference presentation is extended to all Medical Physicists across GenesisCare Australia with the intent of providing a wider audience of specialists to generate questions and feedback, and to motivate the Registrar to deliver a comprehensive and professional presentation. Registrar presentations are recorded and provided on request to persons unable to attend, and Registrars are encouraged to review their recorded presentation for aspects that could be improved. The weekly session prior to EPSM is allocated for any Registrars presenting at the conference either orally or via poster to practice their talks and receive feedback for improvement beforehand.

\section{Lectures by a qualified ROMP}

Lectures or presentations by a qualified ROMP are scattered throughout the National TEAP calendar after accounting for all fortnightly tutorials, Registrar presentations, and event specific sessions. These lecture sessions are evenly allocated amongst the five states, with the Chief Physicists of each state nominating members of their staff to deliver a lecture to the Registrars. Nominated physicists are encouraged to align their presentation with Assessment Criteria directly from the CTG though they are welcome to present or lecture on any topic that would be of interest to the Registrars.

\section{Discussion}

The key to success of this program is endorsement and support from all levels of management. The national weekly TEAP training scheme at GenesisCare has ultimately maintained momentum as a result of continued support from 
physics management from national to state level. Since the program's inception it has been the Chief Physicists of each state who have encouraged and enabled their local staff to contribute to the national sessions. The enthusiasm from staff at the local level to provide training to TEAP Registrars is a key driver to the program's success and has resulted in a sustainable program of quality learning opportunities valued by the Registrars. Clinics are also expected by physics management to facilitate and prioritize Registrar access to the weekly sessions around their busy clinical and training workload. Since the scheme started in May 2019 there has been one tutorial cancellation due to unforeseen circumstances, and a small number of postponements and rearrangements with gaps filled without difficulty by canvassing the qualified network for substitutes. This has ensured a reliable weekly session the Registrars can depend on for a TEAP-related learning opportunity.

A considerable benefit of the shared program is that in smaller clinics, and particularly those with a Registrar on site, it no longer needs to be the small group of qualified ROMPs that shoulders the full workload of TEAP training. Rather, they can leverage the expertise of their network of colleagues to provide some of that training. This connectedness can be of substantial benefit to maintaining momentum and enthusiasm for training, and even increase engagement outside of their TEAP training roles.

There is a reasonable time commitment requested of qualified physicists to deliver a lecture, and even more so for those who host tutorials. While it is a good opportunity to earn ACPSEM CPD points, tutors must spend time developing question lists and expected answers, reviewing all answers submitted by the Registrars, and facilitating the tutorial. As noted, tutors are not required to mark and provide individual feedback to each submission as this would further increase the time commitment and perhaps detract from hosting future tutorials. Furthermore, as discussed, the tutor's primary role is intended to be to guide discussion and to engage the Registrars to actively develop a comprehensive response themselves rather than simply being 'passive receivers' of yet more information. It is therefore the responsibility of the TEAP Registrar to take their learnings from the tutorial discussion to better their responses and to submit their refined responses to their Supervisor for review as evidence toward learning outcomes. After any tutorial or lecture Registrars are encouraged to contact the host with any follow up questions should they not fully comprehend the discussed matter.

The organizing team actively seeks detailed feedback from the Registrars to understand what is working well, to hear their solution-focused suggestions, and any additional ideas and thoughts they may have to increase their overall benefit from the sessions. Feedback is encouraged at any time, and the team formally solicits feedback approximately every 6 months, initially via email but more recently via anonymous online survey. Implementations as a result of Registrar feedback in 2019 included requesting tutors to develop more clinical scenario type questions, asking tutors to include past written exam questions, and releasing question lists 2 weeks before the due date (not 2 weeks before the tutorial). The recent anonymous free-text survey results for 2020 were overwhelmingly positive with respect to the weekly sessions and their usefulness, frequency, variety, and breadth. The results also showed most Registrars used tutorial answers as COMET evidence, with several stating that the weekly sessions were accelerating their progress through TEAP as CTG Assessment Criteria were being explicitly addressed. Suggestions for topics to cover in more detail from the 2020 results included treatment planning, brachytherapy, and $\mathrm{kV}$ superficial given the limited access to these treatment techniques but required demonstration of competence. The organizing team are incorporating these topics into the 2021 calendar with the view to deliver these as hands-on demonstrations where possible. As the majority of Registrars stated their centres do not offer additional scheduled TEAP sessions, the national weekly scheme is addressing the need for a well-structured program that frequently addresses TEAP competencies and is resilient to fluctuating local staff levels and clinical workload. Registrar feedback from the recent survey will inform the structure for the 2021 calendar of sessions; in particular the general desire to maintain the frequency of Registrar presentations, increase the number of lectures, and to provide access to expected answers developed by the tutor. It is from regular Registrar feedback that modifications and improvements to the weekly sessions can be implemented to increase their value to the Registrars.

Since the program commenced a small team of three has been dedicated to organizing and maintaining the weekly training schedule. Keeping this team small has meant more control of the format and consistency of content, with easy communication between the group seeing quick turnarounds for decision-making. The current organizing team spans three states (WA, VIC, NSW) which brings a variety of perspectives to the team regarding challenges affecting Registrars that may be unique to their state. The team also spans all levels within the GenesisCare Physics team. This provides an important range of experience that assists to shape the program and provides an advocacy pathway for the program itself and for the ROMP specialists who contribute to it.

A consideration when designing a program such as this is scalability. While clearly more Registrars attending sessions increases efficiency there is a trade-off regarding the benefit to the Registrars. If the group becomes too large some may not be given opportunity or have confidence to contribute to discussion. Over the duration of the national TEAP 
scheme at GenesisCare Australia the number of Registrars has ranged between 8 and 11 as new Registrars join and senior Registrars qualify. This number has proven amenable to the active participation required during the tutorials, as well as to allocating one day a month for each Registrar to deliver a presentation within the calendar year. If an institution or group of institutions were scaling this model to a much larger group of Registrars then a potential solution would be to split into smaller groups for tutorials based on experience, and to have more than one (shorter) Registrar presentation on the same day.

The journey of developing the national weekly scheme has seen many positives, and some less successful experiences that were used to improve the program. One observation is that there was a natural tendency for tutorial hosts to select the best response to each question, which can favour experienced Registrars. This can demotivate inexperienced or poorer-performing Registrars to submit quality answers and can reduce their opportunities to practice verbalizing medical physics concepts to improve their knowledge or performance. The organizers now explicitly request the host tutor select a response from each Registrar that submits answers. Another lesson was drawn from the inadvertent scheduling of two tutorials on different topics only a week apart. Response quality suffered across both tutorials, and scheduling now is maintained at fortnightly intervals. Scheduling a full year of calendar events ahead of time has proven an effective strategy. During the year then it is a simple matter to remind individuals of their upcoming session. Scheduling all events early in the calendar year gives both physicists and Registrars ample time to prepare for their allotted date, which reduces the risk of cancellation or postponement. From experience, completing the calendar year of events can take a few months to achieve. Another major success was the digital platform and online nature of delivery via videoconference, removing any barriers regarding geographic location of a Registrar. All Registrars have equal access to the learning material and network of physicists regardless of whether they are at a large metropolitan institution, a small satellite centre, or a rural site. With the advent of COVID-19 in early 2020 causing many staff to adjust their working and training arrangements, a national training scheme already executed on a digital platform meant the weekly sessions were entirely uninterrupted as physicists could deliver tutorials or lectures from work or home and Registrars too could attend from either location.

\section{Summary}

Moving to a collaborative approach to deliver training opportunities to TEAP Registrars benefits all persons involved. Registrars have access to a regular weekly learning opportunity that draws directly from the CTG, frequent opportunities to practice verbalizing medical physics concepts, access to a wide network of qualified experts (more than 60 as at February 2021), experience with different teaching styles and expectations, and a wider group of Registrars to connect with and use as a support network for exam preparation and learning. For the Supervisor and local physics team the training workload is eased, and they can better focus on reviewing Registrar evidence and assessing their competencies. For the qualified ROMP network there is access to CPD opportunities via facilitating tutorials, delivering lectures, and attending Registrar presentations. For physics management there is the knowledge that their practice is offering Registrars frequent quality learning opportunities where all staff take responsibility, and Registrar and qualified physicist time is more efficiently and effectively utilised.

Funding Funding for this study is not applicable.

\section{Declarations}

Conflict of interest All authors declare that they have no conflict of interest.

Ethical approval This article does not contain any studies with human participants performed by any of the authors.

\section{References}

1. Australasian College of Physical Scientists and Engineers in Medicine (2017) Training, education and assessment program for medical physics and radiopharmaceutical science. ACPSEM, Sydney

2. International Atomic Energy Agency (2009) Clinical training of medical physicists specializing in radiation oncology, training course series 37. IAEA, Vienna

3. Australasian College of Physical Scientists and Engineers in Medicine (2013) Radiation oncology medical physics clinical training guide v3.6. ACPSEM, Sydney

4. Perkins A (2013) Reflections on TEAP: training for the future of medical physics. Australas Phys Eng Sci Med 36:143-145

5. Royal Australian and New Zealand College of Radiologists (2020) Establishing and sustaining regional and rural radiation therapy centres v2.0. RANZCR, Sydney

6. Wilfert L (2018) Drivers of change. Australas Phys Eng Sci Med 41:357-360

7. Haworth A, Fielding AL, Marsh S, Rowshanfarzad P, Santos A, Metcalfe P, Franich R (2020) Will COVID-19 change the way we teach medical physics post pandemic? Phys Eng Sci Med. https:// doi.org/10.1007/s13246-020-00898-9

Publisher's Note Springer Nature remains neutral with regard to jurisdictional claims in published maps and institutional affiliations. 